\title{
THREE- VERSUS TWO-DIMENSIONAL ELECTRON GAS INJECTION IN RESONANT TUNNELLING
}

\author{
T. Figielski, T. Wosiński, A. MĄKosa \\ Institute of Physics, Polish Academy of Sciences \\ Al. Lotników 32/46, 02-668 Warszawa, Poland \\ M. KANIEWSKa aNd K. REgińSKI
}

Institute of Electron Technology, Al. Lotników 32/46, 02-668 Warszawa, Poland

A small "precursor" of resonance is observed before the main resonance peak in the current-voltage characteristic of double-barrier resonant-tunnelling devices. The competition between the precursor and main-peak current is examined within the temperature range $4.2-400 \mathrm{~K}$. The precursor is interpreted as 3DEG contribution to the resonant tunnelling dominated by a 2DEG injection from a triangular well formed under bias in the emitter spacer layer.

PACS numbers: $73.40 . \mathrm{Gk}, 85.30 . \mathrm{Mn}$

We studied double-barrier resonant-tunnelling structures grown in the Institute of Electron Technology in Warsaw by molecular beam epitaxy on (100)-oriented $n^{+}$-GaAs substrates. They consisted basically of two $5.6 \mathrm{~nm} \mathrm{Al}_{0.4} \mathrm{Ga}_{0.6} \mathrm{As}$ barriers separated by a $5.6 \mathrm{~nm}$ GaAs quantum well. Heavily $n$-doped electrode layers were separated from the barriers by several intermediate layers, in which the donor concentration was gradually decreased, in order to prevent the incorporation of segregated dopant into the active part of the structure during epitaxial growth (Fig. 1). After growth, mesa diodes of $100 \mu \mathrm{m}$ diameter were defined using photolithography and Ar-ion beam etching. Ohmic contacts were made by alloying AuGeNi layers. The diodes exhibit remarkably good electrical parameters: the peak-to-valley current ratio 23 at $80 \mathrm{~K}(3$ at $300 \mathrm{~K})$, and the peak current density $1.2 \mathrm{kA} / \mathrm{cm}^{2}$ at $80 \mathrm{~K}$.

These devices display resonant maxima in the tunnel current versus bias voltage which end in a double-step feature (Fig. 2) similar to that frequently reported in the literature, cf. [1], and distinguishable from a single-step feature, cf. [2]. At each step the apparent current follows the load line of the measuring set. In addition, current-voltage characteristics, $I(V)$, of the devices reveal an uncommon feature: the appearance of a "precursor" of resonance at a bias voltage below that of the main resonance peak (Fig. 2). It is distinctly seen while measuring 


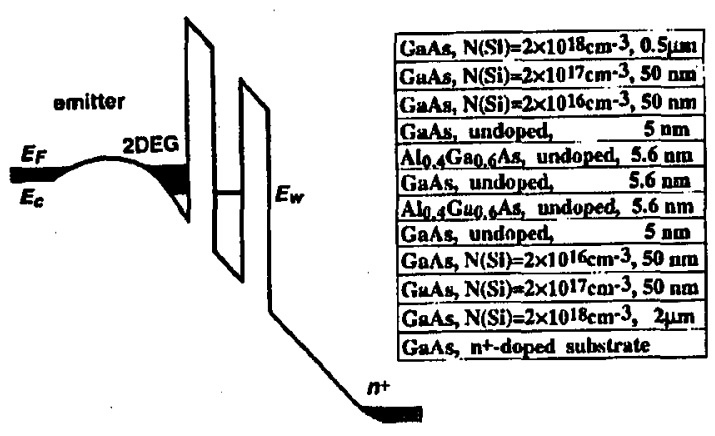

Fig. 1. Spatial variation of conduction-band edge in double-barrier heterostructure having thick spacer layer in emitter region, under bias voltage. On the right hand side: material parameters of the investigated structure.

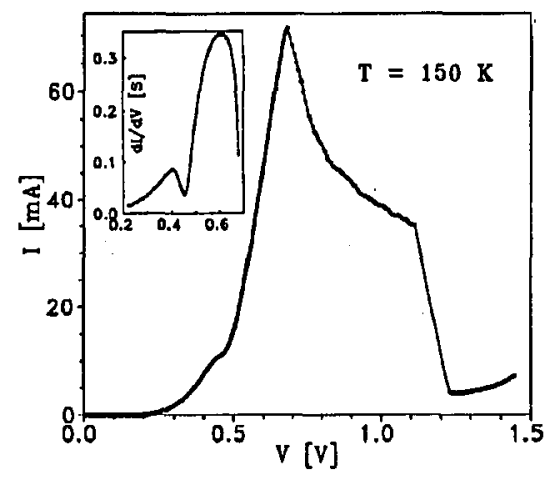

Fig. 2. Current-voltage characteristic of the investigated structure at $150 \mathrm{~K}$. In the inset: differential conductance of the structure in the region of increasing resonant-tunnelling current.

the first derivative of the tunnel current with respect to the applied voltage, as shown in the inset in Fig. 2. The above feature is the principal subject of the present investigations. Similar feature has already been reported in the literature [3-5] but has little been examined in detail.

With undoped spacer layer adjacent to the emitter barrier, the electrons accumulated near the barrier under bias conditions cause a band bending that gives rise to the creation of a quasi-triangular quantum well (Fig. 1). Electron motion perpendicular to the barrier-layer face is then quantized which results in a two-dimensional electron gas (2DEG) occupying a subband within the triangular well. Since $n^{+}$-doped emitter region is separated from the barrier by undoped and lightly-doped layers, an electrostatic bump is formed under bias between this region and the triangular well, as shown schematically in Fig. 1.

In line with the above picture, we propose the following explanation for the resonant part of the $I(V)$ characteristic. The small precursor before the main resonance peak is due to the contribution of ballistic electrons that traverse over 
the bump; this is the 3DEG part of the resonant current. Instead, the resonant tunnelling of electrons occupying the subband in the triangular well gives rise to the main resonance peak. Thus, the source of electrons responsible for the dominant resonant tunnelling is 2DEG.

To verify the above hypothesis, we thoroughly investigated the static $I(V)$ characteristic and $\mathrm{d} I(V) / \mathrm{d} V$ curve within the temperature range $4.2-400 \mathrm{~K}$. Dependences on the reciprocal temperature of three distinctive quantities revealed in the measured characteristics are compiled in Fig. 3. The first one is the main-resonance component of the tunnel current, defined as the difference between the current intensities at the resonance and in the valley beyond the peak. The second is the off-resonance component of the tunnel current measured just in the valley. The third is a measure of the precursor amplitude, defined as the difference between the relevant local maximum and minimum in the $\mathrm{d} I(V) / \mathrm{d} V$ curve, multiplied by the difference of the voltages corresponding to the above extrema (see inset in Fig. 2).

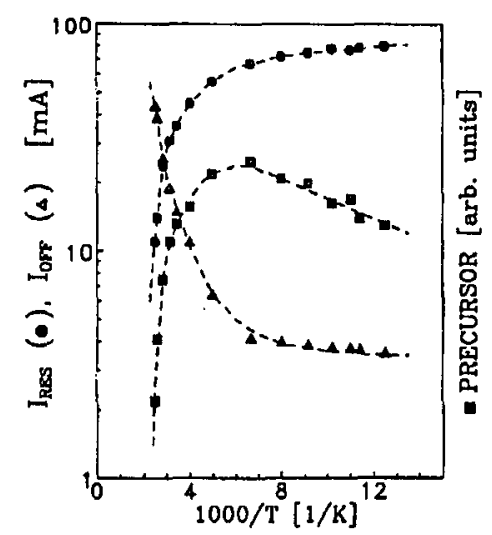

Fig. 3. Resonance, $I_{\mathrm{RES}}$, and off-resonance, $I_{\mathrm{OFF}}$, currents, and precursor amplitude, PRECURSOR, defined in text, versus reciprocal temperature.

As seen in Fig. 3, the resonant component of the tunnel current dominates the off-resonance one at temperatures below $330 \mathrm{~K}$. The off-resonance tunnelling does not conserve in-plane electron momentum, so it is likely LO-phonon assisted process, which is strongly enhanced at higher temperature. In fact, the LO-phonon contribution to the valley current is evident in magneto-tunnelling experiments $[2,4,6]$.

It is significant that below $150 \mathrm{~K}$ the main-resonance component goes up tending to saturation while at the same time the precursor amplitude steadily decreases, showing apparent activation energy of $9 \mathrm{meV}$, and disappears almost completely at $4.2 \mathrm{~K}$. The latter behaviour distinguishes the present precursor from similar features observed at very low temperature which have been due to the tunnelling via donor states in the well [7]. We may assume that the precursor amplitude represents the number of $3 \mathrm{D}$ electrons which surmount ballistically the 
electrostatic bump and are next transmitted resonantly throughout the double barrier. Then, the physical meaning of the observed activation energy would be the difference between the Fermi energy in the emitter region and the energy of the bump top. The electrons surmounting the bump are mainly thermalized in the triangular well but in a small part can reach ballistically the barrier, as evidenced by the proportion between the main peak and precursor amplitude.

We should also keep in mind the difference between the cases of 2DEG and 3DEG injection, which follows from the conservation of total energy and in-plane momentum of an electron in resonant tunnelling. For 2DEG, the electron flux transmitted from the emitter side into the well is a resonant function of the energy difference between the emitter and well subband levels. Instead, for 3DEG, tunnelling begins when the energy difference between the emitter Fermi level, $E_{\mathrm{F}}$, and the subband bottom in the well, $E_{\mathrm{w}}$, becomes positive: $\Delta E \equiv E_{\mathrm{F}}-E_{\mathrm{w}} \geq 0$. Tunnelling electrons are located on a disc intersecting the emitter Fermi sphere at energy $E_{\mathrm{w}}-E_{\mathrm{c}}$, where $E_{\mathrm{c}}$ is the energy of the conduction-band edge in the emitter [8]. As $\Delta E$ increases, there are more tunnelling electrons since the disc area becomes larger. However, at the same time each electron carries less current since the momentum component perpendicular to the interface gets smaller and smaller. Thus, if the transmission coefficient is assumed constant, the resulting current will be proportional to $\Delta E\left(E_{\mathrm{F}}-E_{\mathrm{c}}-\Delta E\right)^{1 / 2}$, and vanishes when $\Delta E \geq E_{\mathrm{F}}-E_{\mathrm{c}}$. Unfortunately, owing to various additional effects, e.g. building up a space-charge in the well, the above features could not be identified in the observed $I(V)$ curves.

This work is supported by the State Committee for Scientific Research (Republic of Poland), grant PBZ-101-01-11.

\section{References}

[1] T. Figielski, A. Mąkosa, T. Wosiński, P.C. Harness, K.E. Singer, Solid State Commun. 91, 913 (1994).

[2] T. Figielski, S.A. Vitusevich, A. Mąkosa, W. Dobrowolski, A.E. Belyaev, T. Wosinski, R.V. Konakova, L.N. Kravchenko, Solid State Commun. 94, 93 (1995).

[3] J.S. Wu, C.P. Lee, C.Y. Chang, K.H. Chang, D.G. Liu, D.C. Liou, J. Appl. Phys. 69, $1122(1991)$.

[4] Y.G. Gobato, F. Chevoir, J.M. Berroir, P. Bois, Y. Guldner, J. Nagle, J.P. Vieren, B. Vinter, Phys. Rev. B 43, 4843 (1991).

[5] M. Buchanan, H.C. Liu, T.G. Powell, Z.R. Wasilewski, J. Appl. Phys. 68, 4313 (1990).

[6] R.E. Pritchard, P.C. Harness, L. Cury, J.C. Portal, B. Khamsehpour, W.S. Truscott, K.E. Singer, Semicond. Sci. Technol. 6, 626 (1991).

[7] M.W. Dellow, P.H. Beton, C.J.G.M. Langerak, T.J. Foster, P.C. Main, L. Eaves, M. Henini, S.P. Beaumont, C.D.W. Wilkinson, Phys. Rev. Lett. 68, 1754 (1992).

[8] S. Luryi, Appl. Phys. Lett. 47, 490 (1985). 